\title{
Dynamic Analysis of an Ethanol Steam Reformer for Hydrogen Production
}

\author{
Omar R. Llerena Pizarro ${ }^{a}$, Carlos Ocampo-Martinez ${ }^{b}$, Senior Member, IEEE, \\ Maria Serra Prat ${ }^{b}$ and José Luz Silveira ${ }^{c}$ \\ ${ }^{a}$ Laboratory of Mechanical Engineering Universidad Politécnica Salesiana (UPS), Cuenca, Ecuador \\ ${ }^{b}$ Universitat Politècnica de Catalunya, Institut de Robòtica i Informàtica Industrial (CSIC-UPC), Barcelona, Spain \\ ${ }^{c}$ Department of Energy, Sao Paulo State University (UNESP), Guaratinguetá, Brazil
}

\begin{abstract}
The attempt to improve hydrogen quality has motivated research focused solely on the design of reactors for hydrogen production, paying little interest to their modelling and much less attention to its dynamic analysis. For these main reasons, this paper is focused on the dynamic analysis of a mathematical model of an ethanol steam reformer (ESR) for producing hydrogen. This analysis differs from other nonlinear analyses since it is applied to a specific seventh-order system, while literature presents analyses for at most fourth-order systems. The nonlinear model used is based on mass balances. It is represented by partial differential equations (PDEs), which are converted into ordinary differential equations (ODEs) by the finite-differences method over the space. A general nonlinear dynamic analysis based on equilibrium points and their local stability is carried out by using these ODEs. Dynamic analysis results show that the studied reactor has a stable equilibrium point for a physical-sense range of inputs (ethanol + water), which allow to have available key criteria for the design of control strategies.
\end{abstract}

\section{INTRODUCTION}

Hydrogen is considered an excellent energy vector with an important role in reducing harmful emissions to the environment when it comes from renewable sources such as ethanol, which is produced from biomass fermentation processes [18]. According to [1], there are three techniques for hydrogen production from ethanol: steam reforming (SR), partial oxidation (POX) and auto-thermal reforming (ATR). According to the same author, the SR technique is the most used due to its high efficiency and lower operating temperature compared to both POX and ATR. In this process, a mixture of fuel (ethanol) and steam is supplied to the reformer. The process is endothermic and then it requires an external heat source. The conversion of the mixture occurs in the presence of a catalyst. The product of the reforming is a flow of gases, containing mainly hydrogen, carbon monoxide, carbon dioxide and methane. The catalyst is able to accelerate the rate of reaction and improve the performance of the reformer. Cobalt-based catalyst has been considered an appropriate choice because of its low cost and high activity, especially to produce hydrogen at a moderate temperature [4], [5].

The mathematical modelling of a catalytic reactor is not a simple and straightforward process. Rather, it is a complex process due to some main factors. Among others, we can mention: (i)
- the nonlinear nature of the ESR,

- the modelling errors induced by its complicated kinetics, and

- load disturbances in the inlet composition.

However, over the last decades great advances in terms of ESR modelling have been achieved. For instance, a complete model (mass and energy balances) of three-stage low-temperature ethanol steam reformer for fuel cell application is presented in [9]. In [10], a controllability analysis over this model was done. Here, the nonlinear model was linearized before applying a controller and, in [13], a linear predictive control was applied. In addition, a rigorous computational model for hydrogen production from bio-ethanol is presented in [3] and some control strategies are applied in [2], [15]. Moreover, a modelling of the bio-ethanol reforming process for the production of hydrogen is presented in [7]. Finally, more recent research shows a mathematical model of an ESR plus a membrane separation for pure hydrogen production [16]. In all these works, linear control techniques were applied. The controller must deal with the high nonlinearities present in the dynamics of the reformer and be able to satisfy the hydrogen demand of the FC by keeping carbon monoxide as low as possible. So far, specialized literature shows a large number of models and their controllers after a linearization procedure. However, to the best of the knowledge of the authors, there are not exist works involving a nonlinear dynamic analysis of the ESR.

Dynamic analysis must be connected directly to mathematical modeling before applying a controller. The main objective of this analysis is to know whether the modelled system is stable or not within a dynamical range of interest according to the working/control specifications afterwards. From the classical stability literature [19], a process is considered as stable if it starts its operation at a initial condition close to the desired point of operation and it remains in this position for that moment on. Different tools for analyzing the dynamical behaviour of a system, corresponding to nonlinear phenomena, have been used in the literature, such as Phase Plane Analysis and Lyapunov (linearization method and direct method) for second-order systems [17], [8], and Normal Form of Fold Bifurcation or Equilibrium Points and their stability for higher 
Reforming Stage

Membrane Separation Stage

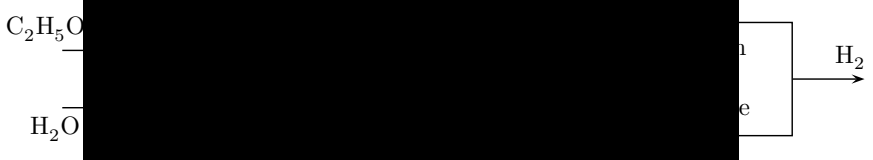

Figure 1: Scheme of the ESR

order systems [11], [14]. Under the light of this brief state of the art, the main contribution of this paper relies on the dynamical analysis of an ESR towards the determination of the stable regios for a simpler control approach. The analysis carried out in this paper differs from all of the aforementioned analyses since they are applied to a specific seventh-order system, while literature presents analysis for lower-order systems.

The remainder of this paper is organized as follows. Section II gives a brief description of chemical reactions of the ESR used in this paper and presents a description of the mathematical model (PDEs), spatial discretization of the nonlinear model and the resultant model based on ODEs. Section III presents the methodology used for the dynamic analysis (equilibrium points and their stability). Next, Section IV shows and discusses the main results of the analysis carried out. Finally, the conclusion and some future works are drawn in Section V.

\section{SYSTEM DESCRIPTION}

In this paper, the ethanol steam reformer analyzed use a cobalt-based catalyst and corresponds to a real laboratory system validated experimentally $[18,19]$. ESR reactions (Eq. (1) to Eq. (4)) over cobalt-based catalyst occur simultaneously within the reformer with the same thermodynamic conditions, and are expressed as follows [6], [20]:

$$
\begin{aligned}
\mathrm{C}_{2} \mathrm{H}_{5} \mathrm{OH} & \longrightarrow \mathrm{CH}_{3} \mathrm{CHO}+\mathrm{H}_{2}, \\
\mathrm{C}_{2} \mathrm{H}_{5} \mathrm{OH} & \longrightarrow \mathrm{CO}+\mathrm{CH}_{4}+\mathrm{H}_{2}, \\
\mathrm{CO}+\mathrm{H}_{2} \mathrm{O} & \longrightarrow \mathrm{CO}_{2}+\mathrm{H}_{2}, \\
\mathrm{CH}_{3} \mathrm{CHO}+3 \mathrm{H}_{2} \mathrm{O} & \longrightarrow 2 \mathrm{CO}_{2}+5 \mathrm{H}_{2},
\end{aligned}
$$

Reaction rates represent how fast the chemical conversion occurs while the detailed expressions and physical explanations for each reaction in (1) can be found in [21]. In this reformer, inlet components are a mixture of the ethanol and water, and outlet components are reformed gases containing mainly hydrogen, carbon dioxide, carbon monoxide and methane as depicted in Figure 1.

\section{A. ESR Mathematical Model}

Some properties of the dynamical model of the ESR are widely studied and discussed in the specialized literature [9], [16]. Here, a brief description of the mathematical model of the catalytic reactor used in this paper is carried out in order to perform the dynamic analysis. This model was proposed and experimentally validated by [16] and it stands for a catalytic reactor. The mass balance is represented by PDEs due to its dependence on the time and length of the reactor as follows:

$$
\begin{aligned}
& \frac{\partial C_{j}(t, z)}{\partial t}+C_{j}(t, z) \frac{\partial v(t, z)}{\partial z}+v(t, z) \frac{\partial C_{j}(t, z)}{\partial z}=\sum_{i} \nu_{j, i} r_{i}(t), \\
& C_{j}(0, z)=C_{j, 0}(z) \quad \forall z \in[0, L], \\
& C_{j}(t, 0)=C_{j, i n}(t) \quad \forall t \geq 0,
\end{aligned}
$$

where (3) and (4) are the initial and the boundary conditions, respectively, while $i \in(a, b, c, d)$ is the reaction index according to (1). Variable $t$ indicates time while $z$ the position along the axial direction of the reactor (in $\mathrm{m}$ ). Notice that $L$ is the axial length of the reactor (in m). $C_{j}$ indicates the concentration of $j$-th component (in $\mathrm{mol} / \mathrm{m}^{3}$ ), and $C_{j, \text { in }}$ the concentration at the reactor inlet. The stoichiometric coefficient of the $j$-th component in the $i$-th reaction (dimensionless) is denoted by $\nu_{j, i}$ while the reaction rate of the $i$-th reaction by $r_{i}$ (in $\mathrm{mol} /\left(\mathrm{m}^{3} \mathrm{~min}\right)$ ). This latter describes the speed of each chemical reaction in (1) and depends jointly on the concentration, temperature, pressure and time through nonlinear relations (see [16] for further details). The linear velocity of the gas mixture is denoted by $v$ (in $\mathrm{mol} / \mathrm{s}$ ) and is defined as $v=v_{\text {in }}(1+\epsilon X)$, where $\epsilon$ represents the molar relation of the considered reaction and $X$ the conversion rate.

\section{B. Spatial Discretization}

In order to convert the PDEs in (2) into a set of ODEs for the dynamic analysis, a spatial discretization is performed. For this purpose, backward-finite differences are applied as follows [16]:

$$
\frac{\partial C_{j}}{\partial z}=\frac{C_{j}(z)-C_{j}(z-1)}{\Delta z} .
$$

The amount and complexity of the ODEs depends directly on the number of volumes in which the ESR is divided. More divisions of volume means larger number of equations. Therefore, in order to obtain a simpler model for the dynamic analysis, the ESR is divided into 10 slices of smaller size and it is assumed that the remaining slides show quite similar behaviour with respect the initial one.

\section{Analyzed ODEs}

The cardinality of the set of ODEs obtained as a result of spatial discretization is 70 (7 concentrations x 10 differential volumes). However, this set of ODEs represents only one unit of volume (seven equations) as depicted in Figure 2.

From the point of view of the dynamic analysis, if it is possible to analyze the stability of one unit, the remaining units will show a similar behaviour by the consideration of homogeneity. Then, the set of ODEs of one unit/slide is presented as

$$
\frac{d x(t)}{d t}=f_{i}(\mathbf{x}(\mathbf{t}), \mathbf{u}(\mathbf{t})),
$$

for all $t \geq 0$, where $\mathbf{x} \in \mathbb{R}^{7}$ is the vector of system states (concentrations for each component in a slide), $\mathbf{u} \in \mathbb{R}^{2}$ is the vector of inputs (reactant inflows) and $f_{i}: \mathbb{R}^{7} \longmapsto \mathbb{R}^{7}$, for 


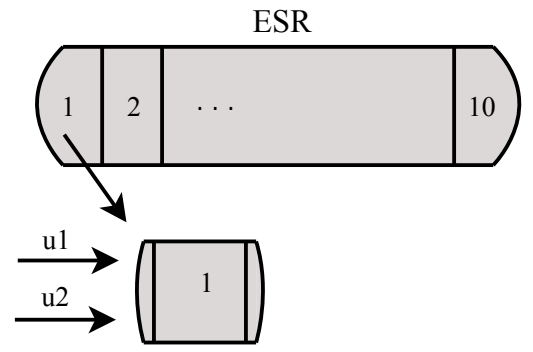

Figure 2: First volumetric unit of the ESR

$i \in\{1, \ldots, 7\}$, are the corresponding nonlineal mappings of the concentrations along the volumetric unit.

\section{Dynamical AnAlysis}

Before applying control techniques, it is quite convenient to know whether or not the system is stable and its stability/instability regions. In this section, a general nonlinear dynamic analysis (based on equilibrium points and Lyapunov stability [19]) is performed over the ODEs in (6).

\section{A. Equilibrium points}

In dynamic analysis, equilibrium points play an important role. These points determine the system status in which the system behaves in steady state. According to the classical theory, to compute the equilibrium points in a system, the following relation must hold:

$$
f_{i}(\mathbf{x}(\mathbf{t}), \mathbf{u}(\mathbf{t}))=\mathbf{0} .
$$

Before solving (7) to find the equilibrium points, the following considerations should be taken into account:

- ESR is operating under normal operation conditions. In other words, concentrations must be non-zero and positive for all $t \geq 0$. Any concentration that does not obey this condition will not be taken into account for the dynamic analysis since there is no physical sense in handling negative or imaginary concentrations.

- After finding the solutions of (7), concentrations $x$ become an equilibrium point, and it is represented by $\mathbf{x}^{\mathrm{eq}}=\left[x_{1}^{\mathrm{eq}}, \ldots, x_{7}^{\mathrm{eq}}\right]^{T}$.

- According to [12], the ESR inputs are physically constrained by lower and upper bound as follows

$$
\begin{aligned}
1.8 \times 10^{-3} & \leq u_{1} \leq 2.4 \times 10^{-3}, \\
8.76 \times 10^{-3} & \leq u_{1} \leq 10.8 \times 10^{-3},
\end{aligned}
$$

where $u_{1}$ represents the molar inflow rate of ethanol while $u_{2}$ is the molar inflow rate of water. Therefore, the nonlinear dynamic analysis is performed taking into account these limitations.

Due to the complexity and nonlinearity of the ODEs (Appendix A), MAPLE is used as computational tool to find out the solutions.



Figure 3: Open-loop system response of the ESR for a given set of inflows

\section{B. Stability analysis}

The equilibrium points determined when solving (7) may be stable or not [14]. First, to determine their stability, the general Jacobian matrix must be computed as follows:

$$
J=\left[\begin{array}{cccc}
\frac{\partial f_{1}(\mathbf{x}, \mathbf{u})}{\partial x_{1}} & \frac{\partial f_{1}(\mathbf{x}, \mathbf{u})}{\partial x_{2}} & \ldots & \frac{\partial f_{1}(\mathbf{x}, \mathbf{u})}{\partial x_{7}} \\
\frac{\partial f_{2}(\mathbf{x}, \mathbf{u})}{\partial x_{1}} & \frac{\partial f_{2}(\mathbf{x}, \mathbf{u})}{\partial x_{2}} & \ldots & \frac{\partial f_{2}(\mathbf{x}, \mathbf{u})}{\partial x_{7}} \\
\vdots & \vdots & \ddots & \vdots \\
\frac{\partial f_{7}(\mathbf{x}, \mathbf{u})}{\partial x_{1}} & \frac{\partial f_{7}(\mathbf{x}, \mathbf{u})}{\partial x_{2}} & \ldots & \frac{\partial f_{7}(\mathbf{x}, \mathbf{u})}{\partial x_{7}}
\end{array}\right]
$$

Some of the inputs of $J$ in (9) are represented in Appendix B. After computing $J$, its eigenvalues $\lambda$ must be computed by using the corresponding characteristic equation [19].

\section{Main Results}

\section{A. Results discussion}

Figure 3 shows the response of the ESR (obtained hydrogen outflow rate) facing initial conditions of both ethanol and water inflow rates. As can be seen, the total ethanol conversion occurs practically in the first five volumetric divisions of the catalytic reactor. From the fifth division on, the inflow rates tend to be constant. In addition, the behaviours of the concentrations are directly related to the reactions presented in (1).

\section{B. Equilibrium points and their stability}

The existence of a stable equilibrium point is necessary to judge the system as stable within a attractive basin. Therefore, Propositions 1 and 2 must hold.

Proposition 1: Consider the model in (6). Assume that the system is operating under normal conditions (positive, nonzero concentrations and constant inlet). Moreover, define the closed set

$$
\mathcal{X}=\left\{\mathbf{x} \in \mathbb{R}^{7}: \mathbf{x}_{\min } \leq \mathbf{x} \leq \mathbf{x}_{\max }\right\},
$$


Table I: Value of the feasible equilibrium point for (6)

\begin{tabular}{c|c}
\hline Coordinate & Value \\
\hline$x_{1}^{\mathrm{eq}}$ & 0.0214823424 \\
$x_{\mathrm{e}_{\mathrm{q}}^{\mathrm{qq}}}$ & 21.126448052 \\
$x_{3}^{\mathrm{qq}}$ & 1.0838471420 \\
$x_{4}^{\mathrm{qq}}$ & 52.743460460 \\
$x_{5}^{\mathrm{eq}}$ & 10.769715900 \\
$x_{6}^{\mathrm{qq}}$ & 10.762420580 \\
$x_{7}^{\mathrm{qq}^{\mathrm{q}}}$ & 0.0066186780 \\
\hline
\end{tabular}

Table II: Values for the eigenvalues of $J$ evaluated in the equilibrium point of Table I

\begin{tabular}{c|c}
\hline Eigenvalue & Magnitude \\
\hline$\lambda_{1}$ & -3.58180044700000 \\
$\lambda_{2}$ & -59216.9862270505 \\
$\lambda_{3}$ & -6344.32219030252 \\
$\lambda_{4}$ & -1891.35422756652 \\
$\lambda_{5}$ & -3.58180047382225 \\
$\lambda_{6}$ & -3.58180010660375 \\
$\lambda_{7}$ & -3.58180100000030 \\
\hline
\end{tabular}

where $\mathbf{x}_{\min } \in \mathbb{R}_{\geq \mathbf{0}}^{\mathbf{7}}$ and $\mathbf{x}_{\max } \mathbb{R}_{\geq \mathbf{0}}^{\mathbf{7}}$ are the vectors of minimum and maximum concentrations, respectively, and $\mathbb{R}_{\geq 0}$ denotes the set of positive real numbers. Therefore, the system (6) must have at least one equilibrium point $\mathrm{x}^{\mathrm{eq}}$ such that $\mathrm{x}^{\mathrm{eq}} \in \mathcal{X} . \diamond$

In order to show the validity of Proposition 1, the system was simulated in a loop that generates no less than 1000 random combinations of inflows (ethanol + water). In all cases, the equilibrium point in Table I was numerically found. As shown in Table I, all concentrations are positive, which shows the existence of a feasible equilibrium point. On the other hand, other numerical values for the solution of (7) were also found but their magnitudes correspond to negative and/or imaginary concentrations.

Proposition 2: Considering the existence of a equilibrium point $\mathrm{x}^{\mathrm{eq}}$ according to Proposition 1. After computing the eigenvalues $\lambda$ of the corresponding Jacobian matrix $J$ in (9), $\mathrm{x}^{\mathrm{eq}}$ is stable if

$$
\operatorname{Re}\left\{\lambda_{i}\left(J\left(\mathbf{x}^{\mathrm{eq}}\right)\right)\right\}<\mathbf{0},
$$

for all $i \in\{1, \ldots, 7\}$, holds.

Once $J$ is stated for the considered ESR under some values of $u_{1}$ and $u_{2}$ satisfying (8), the computation of $\lambda_{i}$ yields in the set of values presented in Table II. From this table and Propositions 1 and 1, it is possible to conclude that the ESR under study shows a unique feasible and stable equilibrium point.

\section{Conclusions}

This paper has presented a numerical approximation of a dynamic analysis for the nonlinear mathematical model of an ethanol steam reformer for hydrogen production. The massbalance model reported in the literature has been used for obtaining the equilibrium points of the system and their stability. Considering that ESR operates under nominal conditions and satisfying its physical limitations (constraints), the performed analysis shows that the system has an unique physicallyfeasible equilibrium point when inflows within their ranges are applied. In addition, the eigenvalues of the corresponding Jacobian matrix of the system evaluated at the yielded equilibrium point have shown that this equilibrium point is stable. Future work will address the analysis of the system including more units of volume and other approaches for numerical stability analysis such as Floquet or Lyapunov Exponents. Also, the analysis of the complete process including the influence of the permeate membrane for hydrogen purification ant the output will be also addressed.

\section{ACKNOWLEDGMENT}

This work has been supported by the project DEOCS (Ref. DPI2016-76493-C3-3-R) from the Spanish Ministry of Economy and Competitiveness. Omar Llerena has been supported by SENESCYT and the Universidad Politécnica Salesiana.

\section{REFERENCES}

[1] L. B. Braga. Aspectos técnicos, econômicos e ecológicos de processos de produção de hidrogênio. $\mathrm{PhD}$ thesis, State University of São Paulo, 2014.

[2] L. Nieto Degliuomini, D. Zumoffen, and M. Basualdo. Plant-wide control design for fuel processor system with $\{\mathrm{PEMFC}\}$. International Journal of Hydrogen Energy, 37(19):14801 - 14811, 2012.

[3] Lucas Nieto Degliuomini, Sebastian Biset, Patricio Luppi, and Marta S. Basualdo. A rigorous computational model for hydrogen production from bio-ethanol to feed a fuel cell stack. International Journal of Hydrogen Energy, 37(4):3108 - 3129, 2012.

[4] M. Dominguez, G. Cristiano, E. López, and J. Llorca. Ethanol steam reforming over cobalt talc in a plate microreactor. Chemical Engineering Journal, 176-177:280 - 285, 2011.

[5] M. Dominguez, E. Taboada, E. Molins, and J. Llorca. Ethanol steam reforming at very low temperature over cobalt talc in a membrane reactor. Catalysis Today, 193(1):101 - 106, 2012.

[6] R. Espinal, A. Anzola, E. Adrover, M. Roig, R. Chimentao, F. Medina, E. López, D. Borio, and J. Llorca. Durable ethanol steam reforming in a catalytic membrane reactor at moderate temperature over cobalt hydrotalcite. International Journal of Hydrogen Energy, 39(21):10902 - 10910, 2014.

[7] Javier A. Francesconi, Miguel C. Mussati, Roberto O. Mato, and Pio A. Aguirre. Analysis of the energy efficiency of an integrated ethanol processor for $\{\mathrm{PEM}\}$ fuel cell systems. Journal of Power Sources, 167(1):151 - 161, 2007.

[8] Javier A. Gallegos and Manuel A. Duarte-Mermoud. On the lyapunov theory for fractional order systems. Applied Mathematics and Computation, 287:161 - 170, 2016.

[9] Vanesa M. Garcia, Eduardo Lopez, Maria Serra, and Jordi Llorca. Dynamic modeling of a three-stage low-temperature ethanol reformer for fuel cell application. Journal of Power Sources, 192(1):208 - 215, 2009.

[10] Vanesa M. Garcia, Maria Serra, and Jordi Llorca. Controllability study of an ethanol steam reforming process for hydrogen production. Journal of Power Sources, 196(9):4411 - 4417, 2011.

[11] Jonathan Hess and Olivier Bernard. Design and study of a risk management criterion for an unstable anaerobic wastewater treatment process. Journal of Process Control, 18(1):71 - 79, 2008.

[12] E. Perez-Contreras, M. S. Prat, C. Ocampo-Martinez, and J. Llorca. Nonlinear model predictive control for hydrogen production in an ethanol steam reformer with membrane separation. In IEEE Conference on Control Applications (CCA), pages 1155-1160, Sept 2016.

[13] D. Recio-Garrido, C. Ocampo-Martinez, and M. Serra-Prat. Design of optimization-based controllers applied to an ethanol steam reformer for hydrogen production. International Journal of Hydrogen Energy, 37(15): 11141 - 11156, 2012.

[14] A. Rincon, F. Angulo, and G. Olivar. Control of an anaerobic digester through normal form of fold bifurcation. Journal of Process Control, 19(8):1355 - 1367, 2009. 
[15] P. Rullo, L. Nieto Degliuomini, M. Garcia, and M. Basualdo. Model predictive control to ensure high quality hydrogen production for fuel cells. International Journal of Hydrogen Energy, 39(16):8635 - 8649, 2014.

[16] Maria Serra, Carlos Ocampo-Martinez, Mingming Li, and Jordi Llorca. Model predictive control for ethanol steam reformers with membrane separation. International Journal of Hydrogen Energy, 42(4):1949 1961, 2017.

[17] Rajneesh Sharma. Lyapunov theory based stable markov game fuzzy control for non-linear systems. Engineering Applications of Artificial Intelligence, 55:119 - 127, 2016.

[18] M. E. Silva. Análise experimental da reforma a vapor de etanol: aspectos técnicos, econômicos e ecológicos. $\mathrm{PhD}$ thesis, Faculty of Engineering of Guaratinguetá, State University of São Paulo, 2010.

[19] Jean-Jacques E Slotine and Weiping Li. Applied nonlinear control. Pearson, Upper Saddle River, NJ, 1991.

[20] M. Torchio, C. Ocampo-Martinez, L. Magni, M. Serra, R. D. Braatz, and D. M. Raimondo. Fast model predictive control for hydrogen outflow regulation in ethanol steam reformers. In American Control Conference (ACC), pages 5044-5049, July 2016.

[21] I. Uriz, G. Arzamendi, E. Lopez, J. Llorca, and L.M. Gandia. Computational fluid dynamics simulation of ethanol steam reforming in catalytic wall microchannels. Chemical Engineering Journal, 167(2-3):603 609, 2011. 